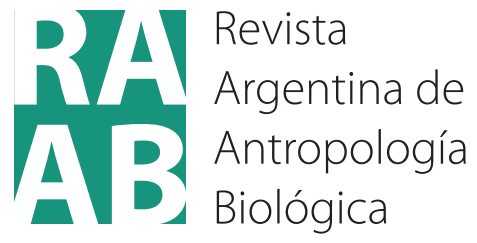

TRABAJO ORIGINAL

\section{Estimaciones de la edad de los individuos, velocidad de locomoción y posible actividad a partir de las huellas humanas del sitio Monte Hermoso 1}

\section{Estimates of age of individuals, locomotion speed and possible activity from human footprints at Monte Hermoso site 1}

\author{
[D Aldo Iván Vassallo
}

1) Laboratorio de Morfología Funcional y Comportamiento, Departamento de Biología e Instituto de Investigaciones Marinas y Costeras, CONICET, Facultad de Ciencias Exactas y Naturales, Universidad Nacional de Mar del Plata, Mar del Plata.

\section{REVISTA ARGENTINA DE ANTROPOLOGÍA BIOLÓGICA \\ Volumen 23, Número 1 Enero-Junio 2021}

Financiamiento: CONICET PIP 2014$2016 \mathrm{~N}^{\circ} 11220130100375$ y Universidad Nacional de Mar del Plata EXA918/18.

*Correspondencia a: Aldo Iván Vassallo, IIMyC-CONICET-UNMdP, Funes 3250,

Mar del Plata (7600). Buenos Aires.

Argentina. E-mail: avassall@mdp.edu.ar

RECIBIDO: 24 de Julio 2020

ACEPTADO: 2 de Marzo 2021

DOI: $10.24215 / 18536387 \mathrm{e} 036$

e-ISSN 1853-6387

https://revistas.unlp.edu.ar/raab

Entidad Editora

Asociación de Antropología Biológica Argentina

\begin{abstract}
Resumen
El sitio arqueológico Monte Hermoso 1 (Buenos Aires, Argentina), presenta un registro abundante de pisadas humanas, datadas $7000 \mathrm{AP}$, que fueron impresas en el sedimento de una marisma mixohalina. El objetivo de este estudio fue realizar estimaciones de edad y velocidad de locomoción correspondientes a 15 rastros de individuos, en base a datos originales de Bayón y Politis (1996). Las estimaciones de edad se realizaron en base a curvas de crecimiento. Las edades estimadas, correspondientes a niños y adolescentes entre 2,5 y 14,5 años, y posiblemente un individuo juvenil-adulto joven, concuerdan con interpretaciones previas. La velocidad de locomoción fue estimada a partir de la relación empírica entre la velocidad adimensional (número de Froude) y la longitud del paso adimensional. Las velocidades obtenidas son heterogéneas, en el rango entre $3-12 \mathrm{~km} / \mathrm{h}$, y se clasifican en: 1) velocidades compatibles con la de caza recolección en niños de edad similar ( $\leq 3 \mathrm{~km} / \mathrm{h}), 2$ ) velocidades compatibles con la caminata de confort, en el rango entre 3,8 y $4,6 \mathrm{~km} / \mathrm{h}$, asociadas a un menor costo energético, quizás de exploración, por parte de niños y adolescentes, del territorio circundante, y 3 ) velocidades $>5 \mathrm{~km} / \mathrm{h}$ compatibles con juego, persecución de una pequeña presa, o huida. La metodología utilizada en este estudio para la estimación de la velocidad puso en evidencia una diversidad de patrones locomotores en los individuos que dejaron sus rastros en el sitio Monte Hermoso 1, lo cual podría mejorar las inferencias sobre los comportamientos asociados. Rev Arg Antrop Biol 23(2), 2021. doi: 10.24215/18536387e036
\end{abstract}

Palabras Clave: huellas humanas, locomoción humana, caza recolección, Holoceno medio, región Pampeana 


\begin{abstract}
The Monte Hermoso 1 archaeological site (Buenos Aires province, Argentina), shows an abundant record of human footprints, dated to $\sim 7000 \mathrm{BP}$, which were imprinted on a mixohaline marsh shores. The objective of this study was to estimate the age and locomotion speed corresponding to 15 tracks of individuals, based on the original data of Bayón \& Politis (1996). Age estimates were made based on growth curves from the literature. The estimated ages, corresponding to children and adolescents between 2.5 and 14.5 years, and possibly 1 juvenile-young adult, generally agree with previous interpretations. The locomotion speed was estimated from the empirical relationship between the dimensionless speed (Froude number) and the dimensionless stride length. Estimated speeds were in the range of $3-12 \mathrm{~km} / \mathrm{h}$ and could be classified into: 1 ) speeds compatible with those of hunting gathering in children of similar age $(\leq 3 \mathrm{~km} / \mathrm{h}), 2)$ speeds compatible with comfort walking, in the range between 3.8 and $4.6 \mathrm{~km} / \mathrm{h}$, associated with a lower energy cost, perhaps for exploration of the surrounding territory, and 3) speeds $>5 \mathrm{~km} / \mathrm{h}$ compatible with play, chasing a small prey, or simply an escape. The methodology used in this study to estimate locomotion speed revealed a diversity of locomotor patterns in the individuals who left their tracks at the Monte Hermoso 1 site, which could improve assessments about associated behaviors. Rev Arg Antrop Biol 23(2), 2021. doi: 10.24215/18536387e036
\end{abstract}

Keywords: human footprints, human locomotion, hunter gatherer, middle Holocene, Pampas region

Los estudios arqueológicos del litoral marítimo de la Región Pampeana comenzaron con los trabajos pioneros de Florentino Ameghino, al cual siguieron numerosos autores que a lo largo del siglo XX propusieron diversas interpretaciones e hipótesis. Una revisión reciente (Bonomo y Scabuzzo, 2017) concluye que el litoral marítimo pampeano sudoccidental fue habitado durante el Holoceno medio y tardío por las mismas poblaciones que habitaron el interior de la región, por lo cual éstas habrían interactuado con distintos ambientes a lo largo del año. Además, propone que en las llanuras próximas a la costa marina los grupos de cazadores-recolectores establecieron campamentos abastecidos con rocas transportadas desde las sierras de Tandilia y Ventania, y con rodados costeros. Desde estos sitios residenciales, estos grupos explotaron los recursos líticos y faunísticos de las zonas de playa y de dunas, dando lugar a los asíllamados talleres de la franja de médanos costeros. El sitio arqueológico Monte Hermoso 1 (Provincia de Buenos Aires, Argentina, $38^{\circ} 59^{\prime} 00^{\prime \prime} \mathrm{S} 61^{\circ} 25^{\prime} 00^{\prime \prime} \mathrm{O}$; Fig. 1), ubicado $6 \mathrm{~km}$ al oeste de la localidad homónima, presenta un registro abundante de pisadas humanas. Si bien los primeros estudios sugirieron que las huellas fueron impresas en las costas de una laguna interduna de agua dulce, un análisis posterior reveló que podría tratarse de los márgenes de una marisma, un ambiente que fue transitado habitualmente por cazadores recolectores durante el Holoceno medio (Blasi, Politis y Bayón, 2013). Gracias a la presencia de restos de animales y vegetales se han podido datar los sedimentos que contienen a las huellas, con fechas entre $7125 \pm 75$ y $6795 \pm 120$ años antes del presente (Bayón y Politis, 1996). Algunas de las preguntas que se han hecho los investigadores respecto a las hueIlas son: ¿Qué actividad o comportamiento es consistente con el patrón que presentan las huellas? ¿Quiénes las dejaron? ¿Cuán cerca de un campamento se encuentra el lugar 
donde fueron registradas las huellas? Las preguntas apuntan a comprender cómo era la relación de las poblaciones humanas con el medio ambiente, cómo era la ocupación del espacio y el uso de los recursos naturales. La ocupación humana del sitio Monte Hermoso 1 fue coincidente con el desarrollo de una llanura de marea en la que se formó una marisma mixohalina (Blasi, Politis, y Bayón, 2013). La interpretación de las huellas humanas allí presentes se ha vinculado con los sitios espacial y temporalmente cercanos La Olla 1 y 2, los cuales funcionaron, según Bayón y Politis (2014), como un campamento transitorio para el procesamiento y consumo de lobos marinos, Arctocephalus australis y Otaria flavescens.

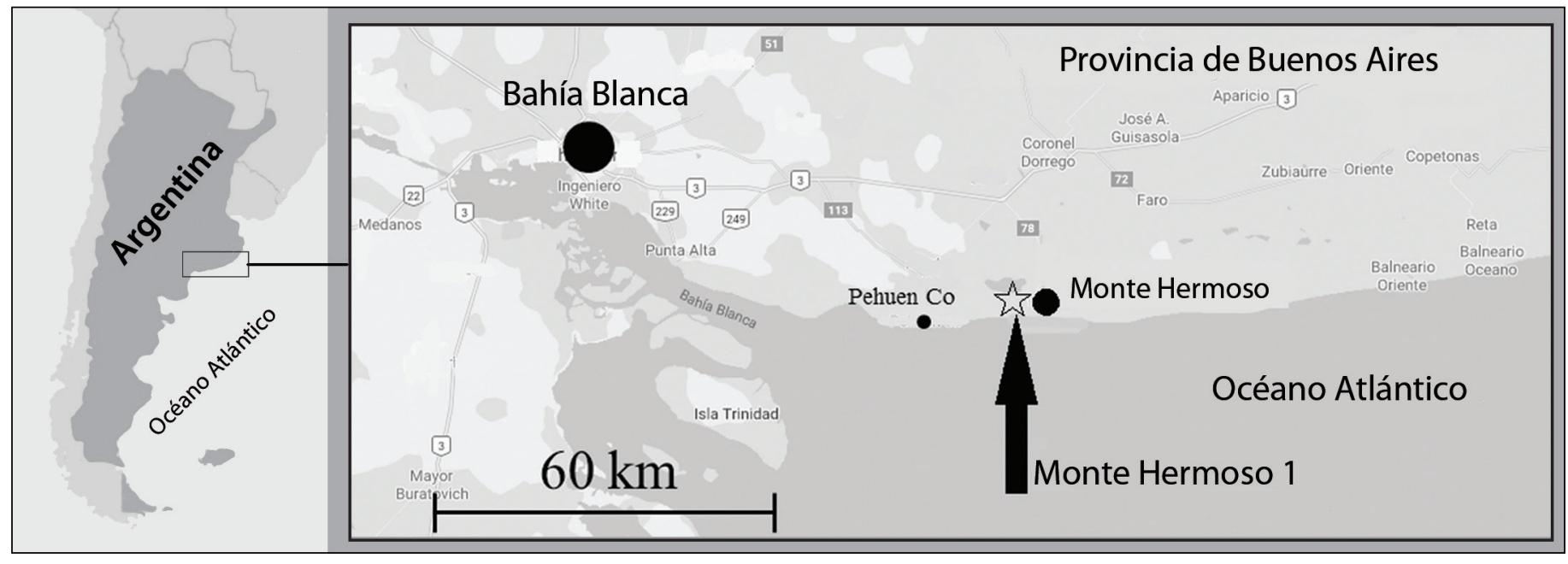

FIGURA 1. Ubicación del sitio Monte Hermoso 1 ( $\left.38^{\circ} 59^{\prime} 00^{\prime \prime} \mathrm{S} 61^{\circ} 25^{\prime} 00^{\prime \prime} \mathrm{O}\right), 6$ kilómetros al oeste de la localidad de Monte Hermoso, sobre la costa, en el sudeste de la Provincia de Buenos Aires, Argentina.

La velocidad a la que se desplaza un individuo al momento de dejar sus huellas puede indicar el tipo de actividad que estaba llevando a cabo. Por ejemplo, una velocidad al caminar de $\sim 4,9 \mathrm{~km} / \mathrm{h}$ (promedio de humanos adultos de ambos sexos, Bohannon y Andrew, 2011) se considera que es la preferida, o confortable, para desplazamientos a media y larga distancia, habiéndose cuantificado experimentalmente que esta velocidad minimiza el costo energético de la locomoción (Ralston, 1958). Sin embargo, la velocidad al caminar puede ser menor que el valor anterior si los individuos se encuentran cazando y/o recolectando (Pontzer et al., 2015), o bien mayor si desean acortar el tiempo de arribo.

Bayón y Politis (1996) efectuaron un detallado relevamiento de huellas humanas (Fig. 2) en un sector del sitio Monte Hermoso 1, incluyendo la longitud de la pisada y del paso, de rastros correspondientes a diferentes individuos, además de proveer información paleoambiental y arqueológica. Estos autores señalan que los rastros están impresos en una superficie isócrona, determinada por la extensión del rastro más largo que cruzó el área de estudio. Por tal motivo ellos consideran que las pisadas fueron dejadas de forma sincrónica, al menos en términos geológicos (Bayón y Politis, 1996). Los autores señalan, además, que las huellas corresponderían a niños, jóvenes y mujeres, aunque no efectuaron estimaciones cuantitativas de la velocidad de locomoción. En el presente trabajo se estima, a partir de los datos publicados en el mencionado estudio, la edad y la velocidad a la cual probablemente se desplazaban los individuos en un sector del sitio Monte Hermoso 1. Se comparan y discuten estos valores con los obtenidos en otros estudios sobre la velocidad de locomoción y su relación con el tipo de actividad llevada a cabo. 


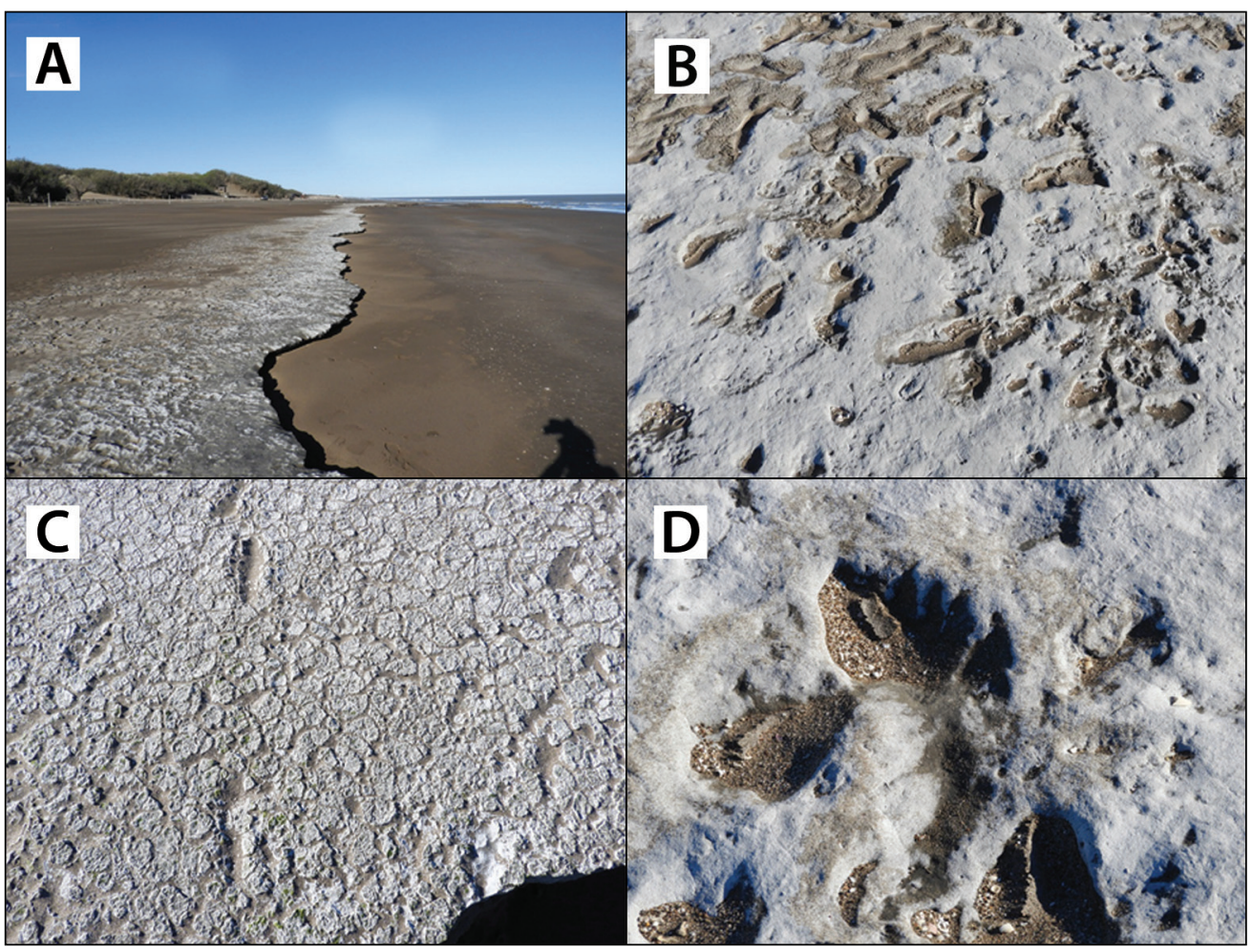

FIGURA 2. Huellas humanas en el sitio arqueológico Monte Hermoso 1. A) Afloramiento de los sedimentos limo fangosos, provenientes de una paleo marisma mixohalina, en cuya costa fueron impresas las huellas humanas hace unos 6800-7100 años A.P. (Bayón y Politis, 1996). Los sedimentos forman actualmente una plataforma de abrasión alargada de $\sim 1 \mathrm{~km}$, ubicada en la parte superior de la playa siguiendo la línea de costa del mar, a $6 \mathrm{~km}$ al oeste de la localidad de Monte Hermoso. B) Huellas humanas. C) Rastros humanos en diferentes direcciones. D) Huella humana de pie derecho (Fotografías del autor).

\section{MATERIALES Y MÉTODOS}

Este estudio se basa en un relevamiento original de 15 rastros, que en conjunto incluyen 76 huellas, en un área de $438 \mathrm{~m}^{2}$. Las estimaciones de edad se realizaron en base al largo total de la huella (Tabla 1 en Bayón y Politis, 1996), utilizando las curvas de crecimiento para niños y jóvenes de Anderson, Blais y Green (1956). La acción erosiva del mar borra a veces parcialmente las huellas por lo que esto pudo haber afectado las mediciones, aunque el efecto es probablemente menor sobre las mediciones de longitud del paso. Estudios previos (Tuttle, Webb, Weidl y Baksh 1990; Bennet y Morse, 2014) muestran que la longitud de las huellas humanas medida en rastros fosilizados puede tanto subestimar como así también sobreestimar el largo del pie. Por tal motivo, y dado que en el trabajo de Bayón y Politis (1996) se proveen varias mediciones de huellas que se atribuyen al mismo individuo, para cada rastro se hizo un promedio de longitud de huella el cual se utilizó para estimar la edad. El dimorfismo sexual en las dimensiones del pie es prácticamente inexistente por debajo de los 12 años por lo que no se llevó a cabo un análisis de este tipo con la presente muestra.

Para la estimación de la velocidad se utilizó la matriz de datos básica publicada como Tabla 1 (Bayón y Politis, 1996), la cual incluye valores del largo total de la huella y la longitud del interpaso, además de otras variables. El método de estimación, basado en la metodología de Alexander $(1976,1984)$ consistió en la aplicación de la relación empírica entre la velocidad adimensional (número de Froude) y la longitud del paso adimensional, según la ecuación: $\lambda / h=\mathrm{a} \cdot\left(v^{2} / g h\right)^{\mathrm{b}}(1)$. Donde $g$ es la aceleración de la gravedad. Conociendo la longitud del paso $\lambda$, que es la distancia entre dos huellas consecutivas del mismo pie (Fig. $3 \mathrm{~A}$ ), medida en rastros fósiles, y estimando el valor de $h$ (la longitud desde la planta del pie hasta 
Revista

Argentina de

Antropología Biológica
TABLA 1. Estimaciones de edad y velocidad correspondientes a los rastros 1 a 15 en base a datos publicados por Bayón y Politis (1996). Los rastros 7 y 11 muestran un posible cambio de la velocidad de locomoción, evidenciado en las diferencias en el largo del paso. El largo del pie en el rastro 1 se estimó a partir de la relación (ancho del pie/largo del pie) obtenida a partir de la muestra total

\begin{tabular}{|c|c|c|c|c|c|c|}
\hline Rastro & $\begin{array}{l}\text { Largo } \\
\text { del pie } \\
(\mathrm{cm})\end{array}$ & $\begin{array}{l}\text { Largo } \\
\text { del paso } \\
(\mathrm{cm})\end{array}$ & $\begin{array}{c}\text { Altura } \\
\text { estimada cadera, } \\
h(\mathrm{~cm})\end{array}$ & $\begin{array}{c}\text { Edad } \\
\text { estimada; } \\
\text { promedio (S.D.) }\end{array}$ & $\begin{array}{l}\text { Número de } \\
\text { Froude } \\
(\lambda / h)\end{array}$ & $\begin{array}{c}\text { Velocidad } \\
\text { estimada } \\
(\mathrm{km} / \mathrm{h})\end{array}$ \\
\hline 1 & 24.6 & 174.4 & 81.9 & $14,5(2,0)$ & 2.1 & 7.18 \\
\hline 2 & 18.5 & 108.2 & 61.7 & $6,4(1,1)$ & 1.7 & 4.1 \\
\hline 3 & 14 & 98.7 & 46.7 & $2,5(0,5)$ & 2.1 & 5.3 \\
\hline 4 & 14 & 104 & 46.7 & $2,5(0,5)$ & 2.2 & 6 \\
\hline 5 & 21.4 & 136.3 & 71.4 & $9,9(1,5)$ & 1.9 & 5.3 \\
\hline 6 & 27.3 & 144.1 & 90.9 & $19,1(2,5)$ & 1.6 & 4.1 \\
\hline 7 & 16 & $85,1 / 132,7$ & 53.3 & $4,0(0,8)$ & $1,6 / 2,5$ & $3,1 / 8,1$ \\
\hline 8 & 21.6 & 131 & 72.1 & $10,1(1,6)$ & 1.8 & 4.8 \\
\hline 9 & 20 & 107.3 & 66.7 & $8,1(1,3)$ & 1.6 & 3.6 \\
\hline 10 & 17.5 & 98 & 58.3 & $5,4(1,0)$ & 1.7 & 3.7 \\
\hline 11 & 18.1 & 129,2 / 91,0 & 60.2 & $6,0(1,1)$ & $2,1 / 1,5$ & $6,3 / 3,0$ \\
\hline 12 & 17 & 94 & 56.7 & $4,9(0,9)$ & 1.6 & 3.5 \\
\hline 13 & 19 & 175 & 63.3 & $7,0(1,2)$ & 2.8 & 10 \\
\hline 14 & 23 & 138 & 76.7 & $12,1(1,8)$ & 1.8 & 4.9 \\
\hline 15 & 21.8 & 200.8 & 72.7 & $10,4(1,6)$ & 2.8 & 11.7 \\
\hline
\end{tabular}

la articulación entre la cadera y el fémur, lo cual representa la longitud funcional de la pierna, (Fig. 3B) puede entonces estimarse la velocidad del individuo cuando dejó ese rastro en particular, despejando la velocidad $v$ de la ecuación; a y b son las constantes de (1). La longitud del paso, definida más arriba, se estimó como (interpaso · 2), dado que la longitud del interpaso es la distancia entre las huellas de los pies derecho e izquierdo. El valor de $h$ se estimó en base a la relación (largo del pie/ $h$ )=0,3 promedio en Homo sapiens, calculado por Raichlen, Pontzer $y$ Sockol (2008). Las constantes a y b se estimaron a partir de registros experimentales de velocidad y longitud del paso obtenidos de tres niños y cuatro adultos (ambos sexos), desplazándose a diferentes velocidades sobre sustrato de arena húmeda en la playa Varese de Mar del Plata. A tal fin, cada uno de los individuos participantes, descalzo, recorrió en varias oportunidades (3-4) una distancia de 25 metros marcada con estacas, con la consigna de hacerlo a diferentes velocidades (caminando, trotando, corriendo) pero manteniendo la velocidad constante durante el recorrido. En cada oportunidad, se cronometró el tiempo (error: 0,01s) en recorrer los $25 \mathrm{~m}$ con la finalidad de obtener la velocidad, y se midió con una cinta métrica (error: $1 \mathrm{~cm}$ ) la longitud de 3 a 5 pasos, calculándose el valor promedio. En cada uno de los individuos participantes se midió la longitud vertical desde el piso hasta la articulación de la cadera ( $h$ ), con una cinta métrica (error: $1 \mathrm{~cm}$ ). Mediante el uso de una planilla de cálculo se efectuó una regresión potencial sobre los datos. La ecuación obtenida fue $y=2.51 x^{0.24}, R^{2}=0,91$ (Fig. 3C) donde $x$ es $v^{2} / g h$, mientras que y es $\lambda / h$ según la ecuación (1). Las velocidades estimadas se clasificaron como: 1) aquellas compatibles con una actividad de caza recolección en niños $(<3 \mathrm{~km} / \mathrm{h}$; Bird y Bird, 2005), 2) compatibles con una caminata a velocidad preferida o de confort (en o cercanas a la franja entre 3,8 y 4,6 km/h; Stansfield et al., 2001) y 3) compatibles con trote o correr (> $5 \mathrm{~km} / \mathrm{h}$ o bien con número de Froude $>2$ ). Dado que el número de Froude representa un valor de velocidad adimensional, permite inferir el tipo de locomoción (caminar vs. trotar o correr) contemplando las diferencias de tamaño corporal entre un niño, un adolescente y un adulto (Alexander, 1976; 1984). 


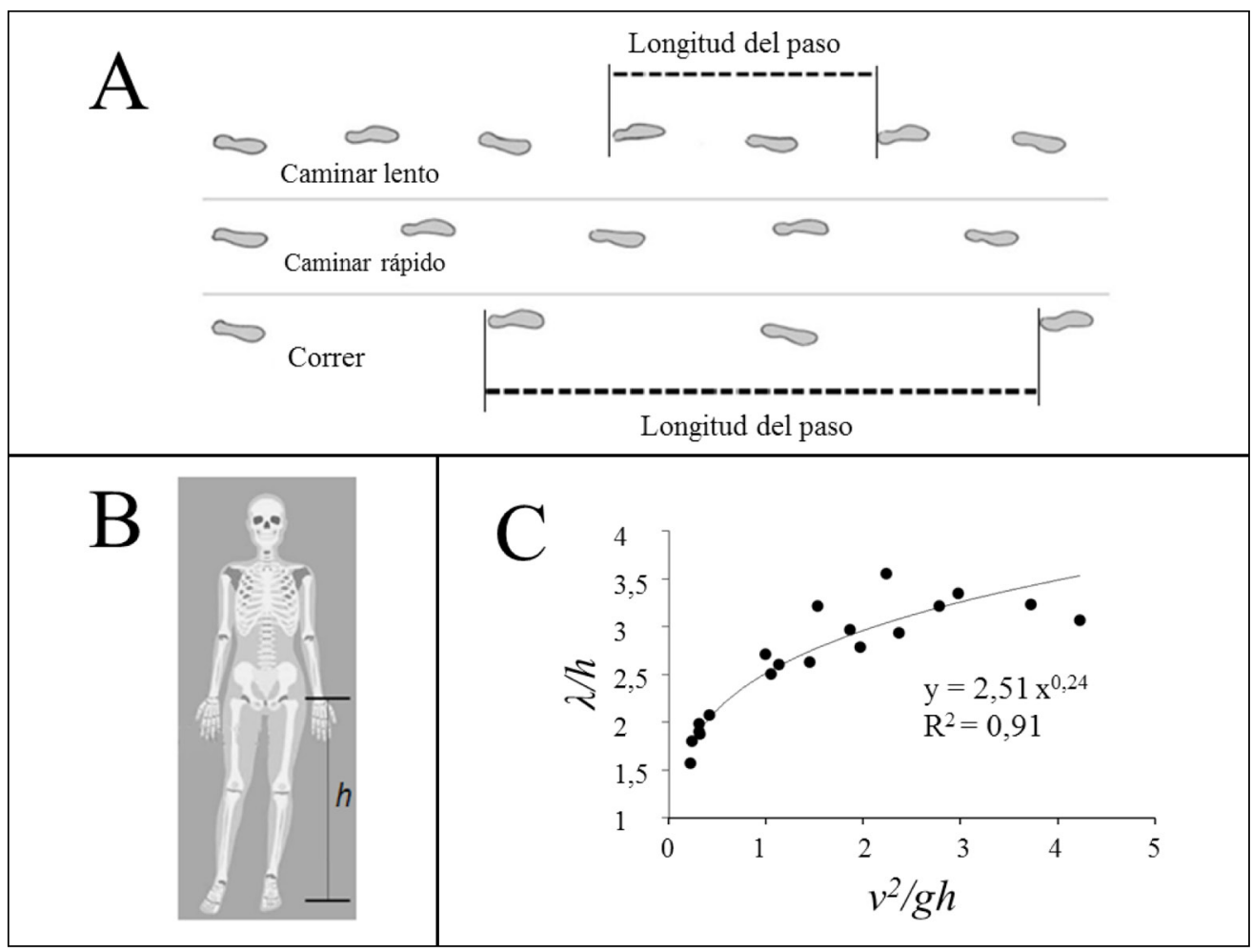

FIGURA 3. A) Longitud del paso ( $\lambda$ ) y su incremento con el aumento de la velocidad. B) Longitud desde la planta del pie hasta la articulación entre la cadera y el fémur (h). C) Relación entre la longitud del paso adimensional $\lambda / \mathrm{h}$ y la velocidad adimensional $\mathrm{v}^{2} / \mathrm{gh}$ ( $\mathrm{g}=$ aceleración de la gravedad) utilizada para las estimaciones de la velocidad de los individuos (basado en Alexander, 1984).

\section{RESULTADOS Y DISCUSION}

La Tabla 1 y Fig. 4 sintetizan los parámetros utilizados para realizar las estimaciones, así como los valores estimados de edad y velocidad. Las edades estimadas de los individuos que dejaron sus huellas están entre los 2,5 y los 14,5 años, salvo el rastro 6 que correspondería a un juvenil-adulto joven, lo que concuerda en general con las edades propuestas por Bayón y Politis (1996). Si bien estos autores mencionan que algunos rastros podrían pertenecer a mujeres adultas, las presentes estimaciones sugieren una mayoría de rastros que corresponderían al rango entre niños y adolescentes. Debe tenerse en cuenta que la inferencia de edad a partir de las dimensiones de las huellas debe realizarse con cautela dado que las dimensiones del pie de los adolescentes se solapan a veces con las de adultos de talla pequeña, especialmente en el caso de las mujeres jóvenes, esto podría estar sucediendo con el rastro 1 (Fig. 4). Por otra parte, las dimensiones del pie pueden variar de acuerdo a la etnia y el nivel de nutrición (Fessler, Haley y Lal, 2005; Stavlas, Grivas, Michas, Vasiliadis y Polyzois, 2005), lo que también puede afectar las estimaciones de edad.

Los valores de velocidad estimados abarcan un rango amplio que va de $\sim 3$ a $\sim 12 \mathrm{~km} / \mathrm{h}$ por lo cual los individuos que dejaron los rastros muy probablemente estaban desarrollando distintos tipos de actividades. Dos rastros podrían estar reflejando un cambio de velocidad, dado que la longitud del paso varía en la misma secuencia de pisadas. Algunas velocidades estimadas son compatibles con una actividad de caza recolección en niños (< $3 \mathrm{~km} / \mathrm{h}$ ), mientas que otras son compatibles con una caminata a velocidad preferida o de confort (en o cercanas a la franja entre 3,8 y 4,6km/h; franja gris en la Fig. 4). Un tercer grupo de velocidades es compatibles con trote o correr (>5-6km/h).

Los rastros 7 y 11 corresponden a niños de edades estimadas entre 3 y 7 años que se 


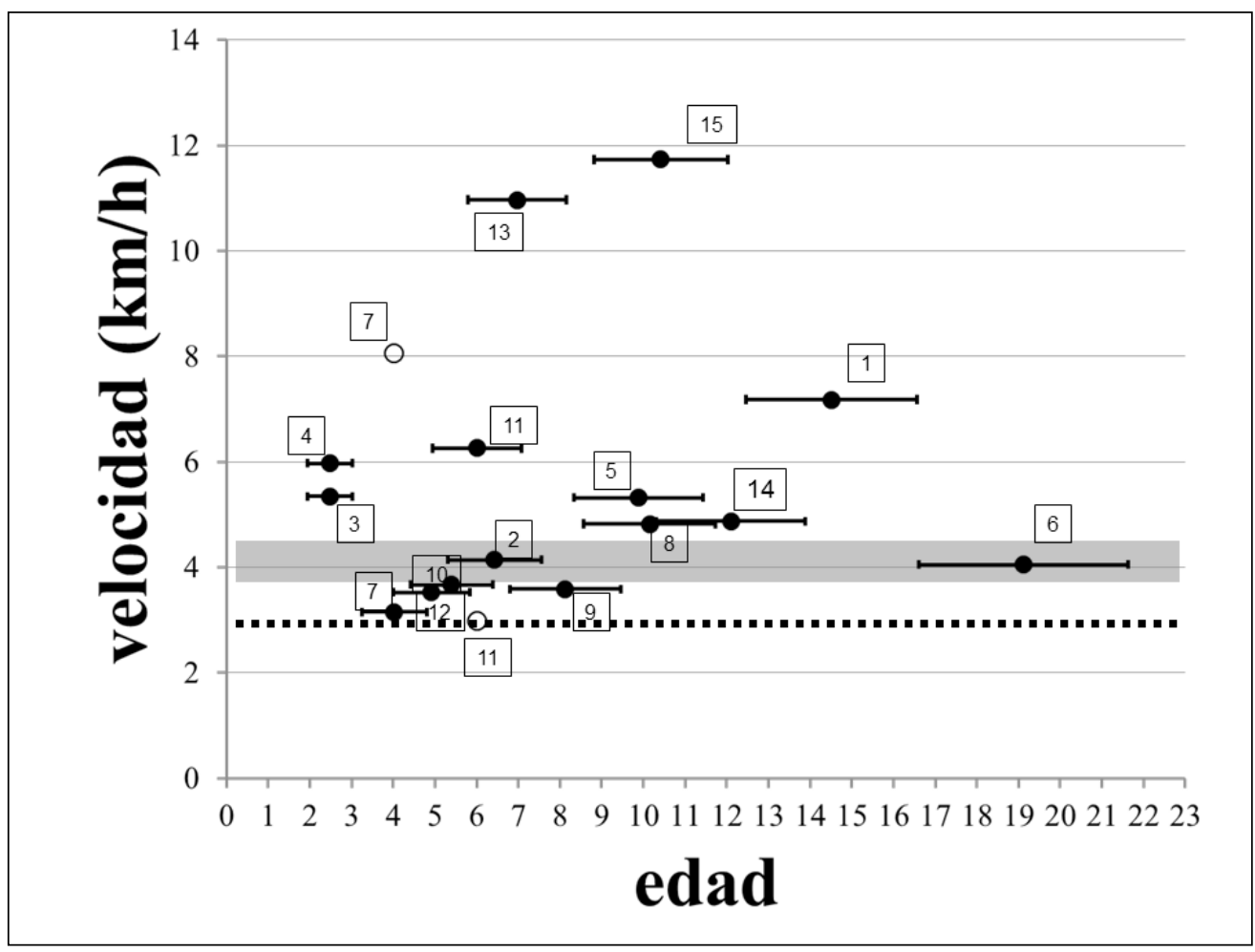

FIGURA 4. Estimaciones de edad (promedio y desvío estándar) y velocidad correspondientes a los rastros 1 a 15 (números en recuadro; Tabla 1), en comparación con la velocidad de locomoción durante la caza y recolección en la etnia Martu (línea punteada, tomado de Bird y Bird, 2005) y con la velocidad de confort al caminar (franja gris, tomado de Stansfield et al., 2001), ambas velocidades en niños de edad similar a las estimadas en el presente trabajo. Los rastros 7 y 11 muestran un posible cambio de la velocidad de locomoción (círculo vacío). El rastro 6 correspondería a un juvenil-adulto joven.

habrían desplazado a velocidades compatibles, en parte de sus rastros, con la actividad de caza recolección. Cierta evidencia apunta a sustentar la participación de niños en esta actividad. En el sitio denominado Arroyo Seco 2 (sudeste de la Prov. de Buenos Aires, Partido de Tres Arroyos) para dataciones correspondientes a 6400AP, se ha encontrado producción material que Politis (1998) interpreta como correspondiente a niños. Este autor considera que una bola de basalto, similar aunque de menor tamaño a las de las boleadoras, recuperada en un enterramiento correspondiente a un niño de corta edad, es una evidencia consistente con la participación de los niños en actividades de juego y/o aprendizaje de caza-recolección. En un estudio realizado con niños de entre 4 y 14 años pertenecientes a la etnia australiana Martu cuyos hábitos alimentarios se basan en la caza y la recolección, se registró una velocidad de $2,86 \mathrm{~km} / \mathrm{h}$ durante actividades que consistieron en la búsqueda, persecución, colecta y procesamiento de frutas, vegetales, insectos, lagartijas, aves y huevos (Bird y Bird, 2005). Se trata de un valor promedio, dado que durante esta actividad hay momentos en que la velocidad puede incrementarse considerablemente al perseguir una presa. Según estos autores, la actividad de caza recolección es realizada por los niños de esta etnia en zonas planas sin pendientes, arenosas, donde puede haber cuerpos de agua transitorios y afloramientos de roca. Por lo tanto, la comparación con las huellas de Monte Hermoso 1 debe hacerse con cautela debido a las posibles diferencias en el sustrato por donde se desplazaban los individuos. Sin embargo, este no parece ser un problema demasiado grave dado que la relación entre la longitud del paso y la velocidad no es apreciablemente diferente entre sustratos duros versus blandos (Fig. 1b en Alexander, 1976). Es interesante señalar que los niños que dejaron los rastros 7 
y 11 probablemente se desplazaron a dos velocidades diferentes, a juzgar por los valores contrastantes de la longitud del paso en dos tramos diferentes de la misma secuencia de pisadas. Así, se habrían desplazado a una velocidad baja de $\sim 3 \mathrm{~km} / \mathrm{h}$, y a velocidades mayores, probablemente trotando (Tabla 1). Las caminatas a velocidades bajas no son una prueba directa de que los individuos estuvieran llevando a cabo una actividad de cazarecolección, sin embargo sí existe evidencia de recolección y uso de productos del mar por poblaciones de la zona durante el Holoceno medio (Politis, Scabuzzo y Tykot, 2009).

Los valores correspondientes a los rastros 2, 9,10 y 12 (rango de edad 4-9 años) están dentro o cercanos al rango de velocidad considerado preferido al caminar en niños de edad similar (Stansfield et al., 2001). La velocidad preferida se refiere a la usada por los individuos al caminar de forma usual y confortable, y correspondería a la velocidad utilizada para desplazamientos a mediana y larga distancia. Debe tenerse en cuenta que la velocidad preferida al caminar varía considerablemente con la edad, por lo que al comparar con las estimaciones de este estudio se recabaron valores experimentales de individuos de edad comparable. Una característica importante de esta velocidad de confort, que posee un significado fisiológico, es que la misma requiere de un menor costo energético. De tal forma, la relación entre costo energético y velocidad al caminar tiene forma de "U" (Ralston, 1958; Selinger, O'Connor, Wong y Donelan, 2015), por lo que velocidades tanto mayores como menores a la de confort poseen un costo energético mayor, como sería el caso del relativamente lento desplazamiento (en promedio, dado que puede haber eventos de locomoción rápida durante una persecución) durante la caza recolección. Los rastros 3, 5, 8 y 14 (rango de edad 2-14 años) también se encuentran cercanos a la velocidad de confort, o preferida, al caminar.

El resto de los rastros indican velocidades similares o mayores a $5 \mathrm{~km} / \mathrm{h}$, a la vez que número de Froude $>2$, consistentes con el trote o la carrera, dos modos de locomoción que poseen en común fases de suspensión en la cual ambos pies se encuentran en el aire, mientras que al caminar siempre hay al menos un pie apoyado en el suelo (Alexander, 1995). Se trataría de velocidades compatibles con diferentes actividades: juego, persecución de una pequeña presa, o simplemente huida. Respecto a las posibles presas que eventualmente pudieran estar persiguiendo, el registro arqueofaunístico para el área (ej. Bayón, Frontini y Vecchi, 2012) sugiere Dasypodidae (mulitas), roedores caviomorfos, peces como Pogonias cromis, el cual podría ser capturado a mano en aguas someras. Un niño cuya edad fue estimada en 2,5 años (rastro 4) trotó o corrió a una velocidad estimada en $6 \mathrm{~km} / \mathrm{h}$ y número de Froude $=2,2$ siendo que a esa edad la velocidad máxima registrada en un estudio fue $9 \mathrm{~km} / \mathrm{h}$. Otro niño de 10 años (rastro 15) corrió a $12 \mathrm{~km} / \mathrm{h}$, edad a la que la máxima velocidad alcanza $20 \mathrm{~km} / \mathrm{h}$ (Schepens, Willems y Cavagna, 1998). El único individuo cuyo rastro, el 6 , puede asignarse a un juvenil-adulto joven se desplazaba a una velocidad estimada de $4,1 \mathrm{~km} / \mathrm{h}$, la cual es inferior a la del caminar confortable en humanos adultos, que es de $\sim 4,9 \mathrm{~km} / \mathrm{h}$ (Bohannon y Andrew, 2011), lo que permite inferir una actividad de recolección.

La dirección de los rastros fue analizada por Bayón y Politis (1996) quienes al no encontrar una orientación predominante, sino más bien una configuración deambulatoria, interpretaron que el área donde fueron impresas las huellas no era una senda de tránsito frecuente.

\section{Consideraciones metodológicas}

Se utilizó el valor de 0,3 promedio en humanos calculado por Raichlen, Pontzer y Sockol (2008) para la relación (largo del pie/h); sin embargo esta proporción puede ser muy variable entre diferentes etnias o incluso entre individuos de una misma población. Consideran- 


\section{AGRADECIMIENTOS}

A Rocío, Lua, Tomás, Matías, Pedro y Kai por su ayuda para la obtención de los datos para efectuar las estimaciones de velocidad. Agradezco a G. Politis, C. Bayón, C. Scabuzzo y a tres revisores anónimos, por sus comentarios sobre el manuscrito.

do un valor de 0,31 en base al desvío proporcionado por los mismos autores, lo cual podría corresponder con las proporciones de individuos pertenecientes a etnias con piernas proporcionalmente más cortas, se obtiene un incremento en la velocidad estimada de $\sim 5 \%$. La ecuación utilizada en el presente estudio se basa exclusivamente en datos obtenidos de seres humanos, presentando un buen ajuste $\left(R^{2}=0,91\right)$, difiriendo en sus parámetros de la obtenida por Alexander (1976; $\left.y=2,3 x^{0.3}\right)$. Esta última incluye datos de diferentes vertebrados además de seres humanos, presentando así una mayor dispersión. La ecuación produce una sobreestimación de la velocidad respecto de los valores estimados en el presente estudio del $\sim 40 \%$, por ejemplo para el rastro 6 arroja $5,8 \mathrm{~km} / \mathrm{h}$. Es necesario señalar que las estimaciones de velocidad realizadas en este estudio no pretenden ser definitivas, ya que son afectadas por varios factores tales como las proporciones corporales de los individuos que las dejaron y la propia preservación de las huellas fósiles. Sin embargo, la heterogeneidad de desplazamientos (caminar lento, rápido, trotar/correr), es un resultado confiable, más allá del refinamiento que pueda lograrse en futuras estimaciones.

\section{CONCLUSIONES}

Las estimaciones de velocidad de este estudio sustentan interpretaciones previas (Bayón y Politis, 1996, 1998; Aramayo, 2009) que sostienen que el área donde fueron dejadas las huellas, los márgenes de una marisma mixohalina, funcionaba como una zona de caza recolección de plantas, aves, huevos y peces por parte de niños y adolescentes, estando los individuos adultos sub representados. Existe evidencia de este tipo de actividades por parte de dicha franja etaria en otras poblaciones de la región (Politis, 1998) así como también de otras áreas geográficas (Bird y Bird, 2005). Sin embargo, algunas de las estimaciones de velocidad serían mayores que las asociadas a la caminata de recolección en niños, y podrían estar relacionadas a caminatas de confort a media o larga distancia, quizás de exploración del área dentro o circundante al sitio, siempre teniendo en cuenta que se trata de niños, por lo que "larga distancia" no implica necesariamente muy lejos de un campamento. Según Bayón y Politis (1996) las huellas del sitio Monte Hermoso 1 se encontrarían a una distancia entre decenas y pocos cientos de metros de un campamento. Otras estimaciones de velocidad de locomoción, aquellas que superan los $5-6 \mathrm{~km} / \mathrm{h}$ o bien un número de Froude $>2$, son compatibles con actividades de juego o bien persecución de pequeñas presas. En su conjunto, los rastros muestran un panorama donde el inicio de actividades de exploración y manutención se superpuso al juego en niños y adolescentes. Estas actividades se habrían efectuado con la vigilancia por parte de pocos individuos jóvenes o adultos, interpretación inferida en base a la sub representación de sus huellas en el área donde se efectuó el relevamiento. La metodología utilizada en este estudio para la estimación de la velocidad puso en evidencia una diversidad de patrones locomotores en los individuos que dejaron sus rastros en el sitio Monte Hermoso 1, lo cual podría mejorar las inferencias sobre los comportamientos asociados.

\section{LITERATURA CITADA}

Alexander, R.McN. (1976). Estimates of speeds of dinosaurs. Nature, 261, 129-130. doi:10.1038/261129a0

Alexander, R. McN. (1984). Stride length and speeds for adults, children, and fossil hominids. American Journal of Physical Anthropology, 63, 23-27. doi:10.1002/ajpa.1330630105

Alexander, R. McN. (1995). Simple models of human movement. Applied Mechanics Reviews, 48, 461-470. doi:10.1115/1.3005107 
Anderson M., Blais, M., Green, W. T. (1956). Growth of the normal foot during childhood and adolescence; length of the foot and interrelations of foot, stature, and lower extremity as seen in serial records of children between 1-18 years of age. American Journal of Physical Anthropology, 14(2), 287-308. doi:10.1002/ajpa.1330140221

Aramayo, S. A. (2009). A brief sketch of the Monte Hermoso Human Footprint Site, South Coast of Buenos Aires Province, Argentina. Ichnos: An International Journal for Plant and Animal Traces, 16, 49-54. doi:10.1080/10420940802470748

Bayón, C. y Politis, G. (1996). Estado actual de las investigaciones en el sitio Monte Hermoso 1 (Provincia de Buenos Aires). Arqueologia, 6, 83-115.

Bayón, C. y Politis, G. (1998). Pisadas humanas prehistóricas en la costa pampeana. Ciencia Hoy, 8(48), 12-20.

Bayón, C., Frontini, R. y Vecchi, R. (2012). Middle Holocene settlements on coastal dunes, southwest Buenos Aires Province, Argentina. Quaternary International, 256, 54-61. doi:10.1016/j. quaint.2011.07.014

Bayón, C. y Politis, G. (2014). The intertidal zone site of La Olla. Early-Middle Holocene human adaptation on the Pampean coast of Argentina. En A. Evans, J. Flatman, y N. Flemming (Eds.), Submerged Prehistoric Archaeology: How Climate Change and Technology Are Rewriting History (pp. 115-130). New York, Estados Unidos: Springer.

Bennett, M. R., y Morse, S. A. (2014). Human footprints: Fossilised locomotion? Dordrecht, Holanda: Springer International Publishing. doi:10.1007/978-3-319-08572-2

Bird, D. W., y Bird, R. B. (2005). Martu children's hunting strategies in the Western Desert, Australia. En B. S. Hewlett y M. E. Lamb (Eds.), Hunter-gatherer childhoods evolutionary, developmental, and cultural perspectives (pp. 129-146). London, Inglaterra: Aldine Transaction. doi:10.4324/9780203789445-9

Blasi, A., Politis, G, Bayón, C. (2013). Palaeoenvironmental reconstruction of La Olla, a Holocene archaeological site in the Pampean coast (Argentina). Journal of Archaeological, 40, 1554-1567. doi:10.1016/j.jas.2012.09.016

Bohannon, R. W., y Williams Andrews, A. (2011). Normal walking speed: a descriptive meta-analysis. Physiotherapy, 97(3), 182-9.

Bonomo, M., y Scabuzzo, C. (2017). Cazadores-recolectores prehispánicos del sudeste del litoral marítimo pampeano. En J. Athor y C. E. Celsi (Eds.), La costa atlántica de Buenos Aires. Naturaleza y patrimonio cultural (pp. 66-86). Buenos Aires, Argentina: Fundación de Historia Natural Félix de Azara.

Fessler, D. M. T., Haley, K. J. y Lal, R. D. (2005). Sexual dimorphism in foot length proportionate to stature. Annals of Human Biology, 32(1), 44-59. doi:10.1080/03014460400027581

Pontzer, H., Raichlen, D. A., Wood, B. M., Emery Thompson, M., Racette, S. B., Mabulla,... Marlowe, F. W. (2015). Energy expenditure and activity among Hadza hunter-gatherers. American Journal of Human Biology, 27(5), 628-637. doi:10.1002/ajhb.22711

Politis, G. (1998). Arqueología de la infancia. Una perspectiva etnográfica. Trabajos de Prehistoria, 55, 5-19. doi:10.3989/tp.1998.v55.i2.300

Politis, G., Scabuzzo, C. y Tykot, R. H. (2009). An approach to pre-hispanic diets in the Pampas during the Early/Middle Holocene. International Journal of Osteoarchaeology, 19, 266-280. doi:10.1002/ oa. 1053

Raichlen, D. A., Pontzer, H., y Sockol, M. D. (2008). The Laetoli footprints and early hominin locomotor kinematics. Journal of Human Evolution, 54(1), 112-117. doi:10.1016/j.jhevol.2007.07.005

Ralston, H. J. (1958). Energy-speed relation and optimal speed during level walking. Internationale Zeitschrift für Angewandte Physiologie, Einschliesslich Arbeitsphysiologie, 17, 277-283. doi:10.1007/ BF00698754

Schepens, B., Willems, P. A., y Cavagna, G. A. (1998). The mechanics of running in children. Journal of Physiology, 509, 927-940. doi:10.1111/j.1469-7793.1998.927bm.x 
Selinger, J. C., O'Connor, S. M., Wong, J. D., y Donelan, J. M. (2015). Humans can continuously optimize energetic cost during walking. Current Biology, 25, 2452-2456. doi:10.1016/j.cub.2015.08.016

Stansfield, W., Hillman, S. J., Hazlewood, M. E., Lawson, A. A., Mann, A.M., Loudon, I. R.,... Robb, J. E. (2001). Normalized speed, not age, characterizes ground reaction force patterns in 5-to 12-yearold children walking at self-selected speeds. Journal of Pediatric Orthopaedics, 21(3), 395-402. doi:10.1097/01241398-200105000-00026

Stavlas, P., Grivas, T. B., Michas, C., Vasiliadis, E., y Polyzois, V. (2005). The evolution of foot morphology in children between 6 and 17 years of age: a cross-sectional study based on footprints in a Mediterranean population. The Journal of Foot and Ankle Surgery, 44(6), 424-288. doi:10.1053/j. jfas.2005.07.023

Tuttle, R., Webb, D., y Weidl, E., y Baksh, M. (1990). Further progress on the Laetoli trails. Journal of Archaeological Sciences, 17, 347-362. doi:10.1016/0305-4403(90)90028-4 